\title{
The Joint Angle Tool for Intraoperative Assessment of Coronal Alignment of the Lower Limb
}

\author{
Ahmed A-H Abood ${ }^{1}$, Juozas Petruskevicius ${ }^{2}$, Björn Vogt ${ }^{3}$, Adrien Frommer ${ }^{4}$, Robert Rödl ${ }^{5}$, Jan Duedal Rölfing ${ }^{6}$
}

\begin{abstract}
Aim: Presentation of the joint angle tool (JAT), a low-cost goniometer for intraoperative assessment of the lower limb alignment.

Background: Intraoperative assessment of coronal alignment is important when performing corrective osteotomies around the knee and ankle, limb lengthening, and trauma surgery. JAT provides surgeons with information about the anatomic and mechanical axes intraoperatively based on true anteroposterior radiographs.

Technique: JAT consists of pre-printed joint orientation angles of the anatomic and mechanical axis including normal variations on a plastic sheet. It is placed on the screen of the image intensifier after obtaining a true anteroposterior image. The pre-printed joint orientation angles can assist the surgeons intraoperatively in achieving the pre-planned axis correction. Here, its feasibility is demonstrated in four cases.

Conclusion and clinical significance: JAT is a modified goniometer that allows intraoperative assessment of the mechanical and anatomic axis. JAT is applicable throughout the entire surgical procedure irrespective of the method of internal fixation and may provide additional reassurance of correct alignment. JAT consists of a plastic sheet with printed joint orientation angles and their normal variation. JAT is freely available from profeedback.dk/JAT/JAT.pdf for use and modification according to the Creative Commons license (CC BY-SA 4.0) if this paper is attributed.

Keywords: Anatomic axis, Bone transport, Deformity correction, Distal femoral osteotomy, Fitbone, High tibial osteotomy, Limb lengthening, MAD, Mechanical axis, NuVasive, Orthopaedic reconstruction, Osteotomy, Technique.

Strategies in Trauma and Limb Reconstruction (2020): 10.5005/jp-journals-10080-1511
\end{abstract}

\section{BACKGROUND}

Intraoperative assessment of the coronal alignment of the lower limb is important in trauma surgery, limb lengthening, and reconstruction surgery. Meticulous preoperative planning based on deformity analysis is essential to achieve the desired alignment postoperatively. Deformity analysis includes measurements of the mechanical axis deviation (MAD), anatomic and mechanical lateral distal femoral angle (aLDFA, mLDFA), medial proximal tibia angle (MPTA), and lateral distal tibia angle (LDTA). ${ }^{1-4}$ The optimal choice of the surgical implant, i.e., intramedullary nail with blocking screws, plate, or external fixator depends on the status of the growth plate, location and severity of the deformity including the need for multiplanar corrections, concomitant limb length discrepancy (LLD), and not least the preference of the surgeon..$^{5-8}$

The reverse planning method described by Baumgart is commonly applied when correcting coronal deformities with intramedullary nails. ${ }^{7}$ Here, the distance from the joint line is the most important intraoperative measure to ensure the correct execution of the preoperative plan. Furthermore, visualisation of the planned nail within the intramedullary canal guides the surgeon. The differences between the mechanical and anatomic axes combined with the limited fluoroscopic field of view make it difficult to assess the correction precisely. Various methods have been described to estimate the mechanical axis intraoperatively, for example, a radiopaque grid underneath the patient, a solid rod, or a cautery cable spanning from the center of the femoral head to the center of the ankle joint under fluoroscopy. ${ }^{7-9}$ Using an intramedullary nail these options are only available at the end of the surgery after the axis has been aligned, for example, the nail has been inserted. All these tools rely on MAD measurements.

\begin{abstract}
1,6Department of Orthopaedic Reconstruction, Aarhus University Hospital, Aarhus, Denmark; Institute of Clinical Medicine, Aarhus University Hospital, Aarhus, Denmark; Danish Paediatric Orthopaedic Research, Aarhus University Hospital, Aarhus, Denmark

${ }^{2}$ Department of Orthopaedic Reconstruction, Aarhus University Hospital, Aarhus, Denmark

${ }^{3-5}$ Children's Orthopaedics, Deformity Reconstruction and Foot Surgery, University Hospital Münster, Münster, Germany

Corresponding Author: Jan Duedal Rölfing, Department of Orthopaedic Reconstruction, Aarhus University Hospital, Aarhus, Denmark; Institute of Clinical Medicine, Aarhus University Hospital, Aarhus, Denmark; Danish Paediatric Orthopaedic Research, Aarhus University Hospital, Aarhus, Denmark, Phone: +45 78450000, e-mail: jan.roelfing@clin.au.dk

How to cite this article: Abood AA-H, Petruskevicius J, Vogt B, et al. The Joint Angle Tool for Intraoperative Assessment of Coronal Alignment of the Lower Limb. Strategies Trauma Limb Reconstr 2020;15(3):169-173.
\end{abstract}

Source of support: Nil

Conflict of interest: None

First, the precision and reproducibility of angles are always better compared with distances. Secondly, weight-bearing significantly influences MAD but does not change joint orientation angles. ${ }^{10}$ Advanced intraoperative fluoroscopic navigation software, patientspecific 3D cutting guides, and hexapod-assisted surgery can also be applied. ${ }^{11-13}$

This technical note presents the joint angle tool (JAT), a simple and low-cost goniometer, visualizing the coronal joint orientation angles during every stage of the surgery. 

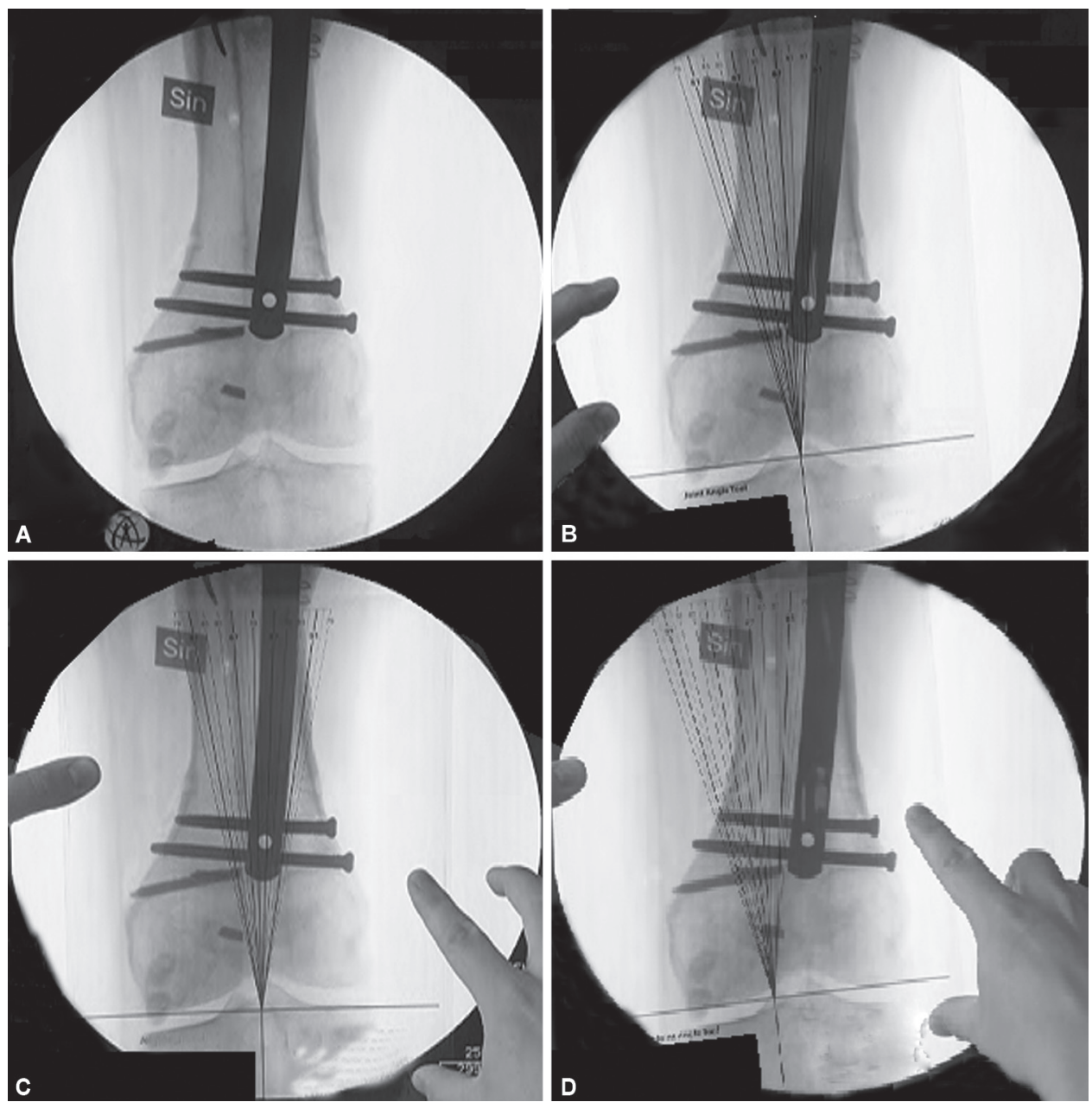

Figs. 1A to D: Examples of applying and adjusting the JAT intraoperatively at the end of a complex surgical procedure illustrate pearls and pitfalls. A prerequisite for correct usage is a true anteroposterior radiograph with the patella facing forward (A). Subsequently, the theatre nurse places the JAT on top of the screen of the image intensifier aiming for tangential alignment of the horizontal line with the femoral condyles and the vertical line intersecting the pre-planned anatomic axis joint center distance (aJCD) (B). In the lower panels, the need for further adjustment to obtain a better rotational (C) and translational alignment (D) of the tool is evident. However, even a subtle misalignment of the tool should be corrected (B)

\section{TECHNIQUE}

JAT consists of a transparent plastic sheet with printed joint orientation angles of the anatomic and mechanical axes and their normal variation: aLDFA $=81^{\circ}(79-83), \mathrm{mLDFA}=87^{\circ}(85-90)$, MPTA $=87^{\circ}(85-90)$, and LDTA $=90^{\circ}(86-92)$. JAT is thus a modified goniometer for the intraoperative assessment of the anatomic and mechanical axis.

Intraoperatively the radiographic assistant or theatre nurse places the JAT on the screen of the image intensifier after the surgeon has obtained a true anteroposterior view of the knee or ankle joint (Fig. 1). The horizontal line of the tool should align perfectly with the femoral condyles/tibial plateau/ankle joint, and the vertical line of the tool should intersect with the inserted guidewire at the joint line (Fig. 3).

Accurate placement is important as it lies in the nature of a straight intramedullary device to redefine the anatomic axis.

The joint should ideally be centered on the screen to minimise distortion by the image intensifier where straight objects tend to appear S-shaped. ${ }^{14}$ Moreover, the joint should be as close to the detector as possible. This serves two purposes, first, it reduces the radiographic distortion, and secondly, it increases the field of view which is helpful to estimate joint orientation angles and anatomic axes more accurately. Before applying the JAT it is also advisable to rotate the image on the image intensifier until the joint line is horizontal.

The editable Adobe Illustrator file (JAT.pdf) is available as Supplementary Digital Content 1 and the Adobe Illustrator file (JAT.ai) is available for free from the homepage: profeedback.dk/ JAT/JAT.ai. The JAT can be freely used, shared, and adapted as long as this paper is cited according to the Creative Commons license: Attribution-ShareAlike 4.0 (CC BY-SA 4.0).

Here, we present four cases demonstrating the clinical applicability of JAT.

Case 1 (Figs 1 and 2): A 32-year-old male presented with a diaphyseal non-union of the left femur four years after a skiing accident (insideout femoral fracture). Hardware failure with protrusion of the primary antegrade nail into the tibia and subsequent failed retrograde exchange nailing preceded the current operation. Genu varum 

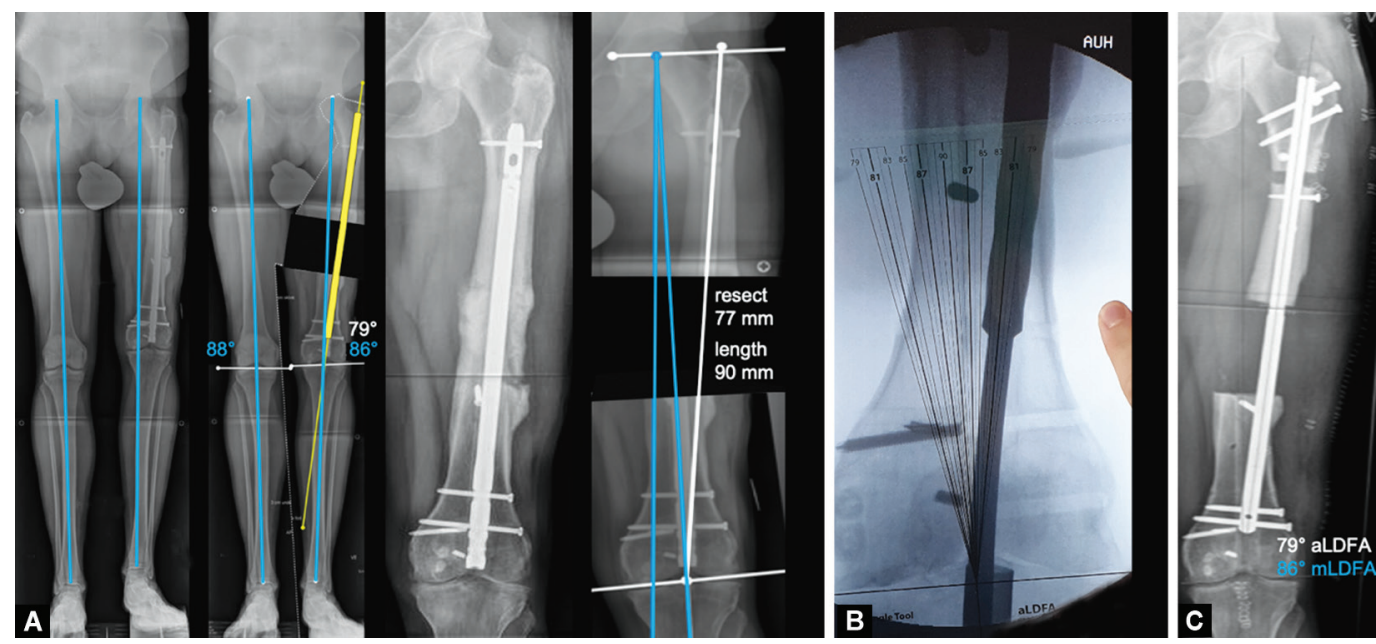

Figs. 2A to C: Bone transport due to non-union. (A) Preoperative planning of $77 \mathrm{~mm}$ femoral bone resection, axis correction, acute lengthening, and $9 \mathrm{~cm}$ bone transport; (B) Intraoperative anatomic axis control using JAT. (C) Postoperative documentation of the accuracy of the planned correction. aLDFA, anatomic lateral distal femoral angle; mLDFA, mechanical lateral distal femoral angle

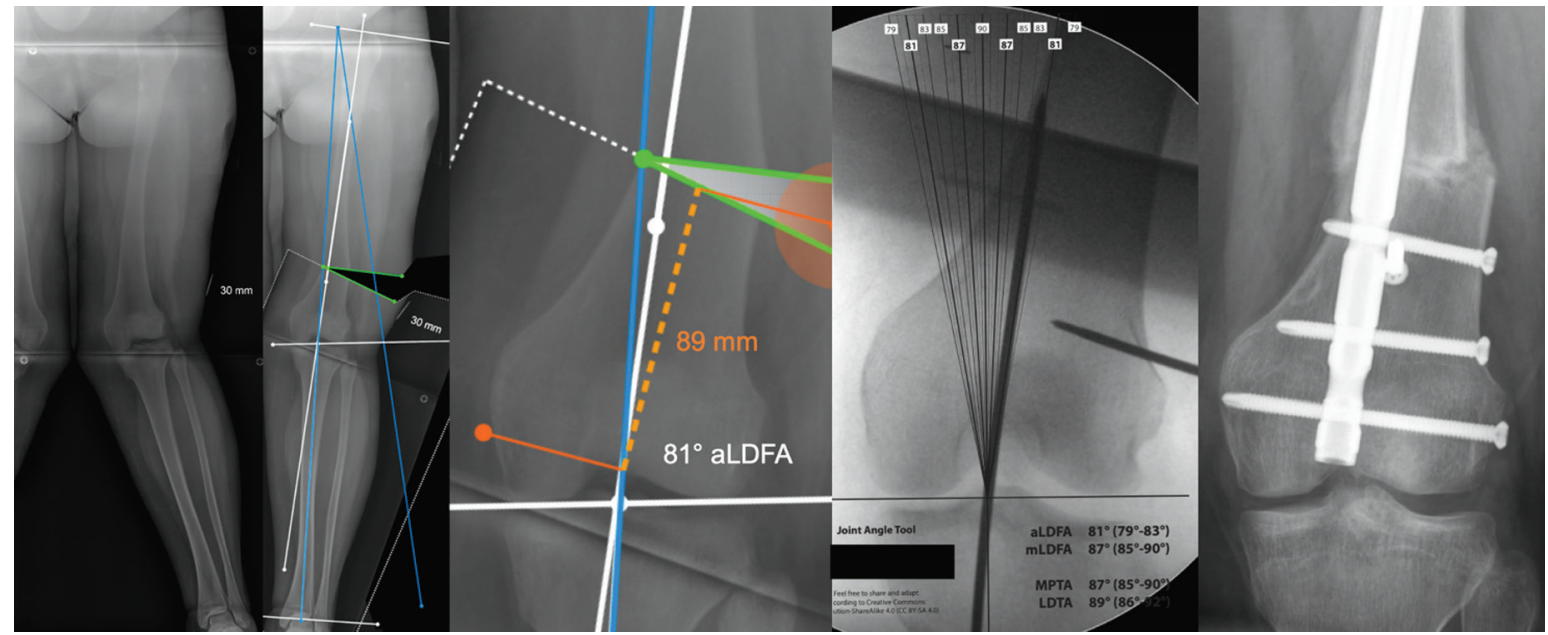

Fig. 3: Distal femoral osteotomy using a retrograde intramedullary nail. aLDFA, anatomic lateral distal femoral angle

$(M A D=26 \mathrm{~mm})$, mild posttraumatic osteoarthritis of the medial compartment of the knee, and $28 \mathrm{~mm}$ LLD were evident (Fig. 2A). Revision surgery included hardware removal, pseudarthrosis resection $(77 \mathrm{~mm})$, acute lengthening, and aLDFA/MAD correction utilising an intramedullary Precice ${ }^{\circledast}$ Bone Transport nail, NuVasive, San Diego, CA, USA. The JAT was applied throughout the entire surgery, that is when inserting a retrograde guidewire, reaming the new canal in the distal femur (Fig. 2B), and after insertion of the antegrade nail. Thereby, the surgeons could achieve and monitor the pre-planned aLDFA of $79^{\circ}$ realigning the limb and offloading the medial compartment of the knee (Fig. 1 and 2C).

Case 2 (Fig. 3): A 14-year-old girl presented with severe genu valgum (MAD $=49 \mathrm{~mm}$ ), $20^{\circ}$ femoral maltorsion and severe medial knee pain, and periodic inability to fully weight bear. The JAT was applied during distal femoral osteotomy and fixation with a retrograde femoral nail (Expert R/AFN, Synthes GmbH, Oberdorf, Switzerland).
Case 3 (Fig. 4): A 17-year-old girl presented with severe genu varum (MAD $=59 \mathrm{~mm}$ ) and $25^{\circ}$ maltorsion of the tibia due to hypophosphatemic rickets. Deformity correction of the right proximal tibia was planned using an intramedullary trauma nail (T2 tibia nail, Stryker Trauma GmbH, Schoenkirchen, Germany) and an osteotomy $60 \mathrm{~mm}$ below the joint line. The intraoperative radiographs demonstrate the ability of the JAT to achieve and monitor the pre-planned angular correction throughout every step of the procedure.

Case 4 (Fig. 5): A 43-year-old male presented with a posttraumatic juxta-articular $9^{\circ}$ valgus deformity of the distal tibia, distal tibiofibular synostosis, and osteoarthritis of the ankle as sequelae of a car accident five years ago. He complained about ankle pain and instability. As the ankle showed an excellent range of motion and the patient refused arthrodesis or joint replacement, a supramalleolar closing wedge osteotomy was performed with a lag screw $(5.5 \mathrm{~mm}$ Large Qwix, Integra 


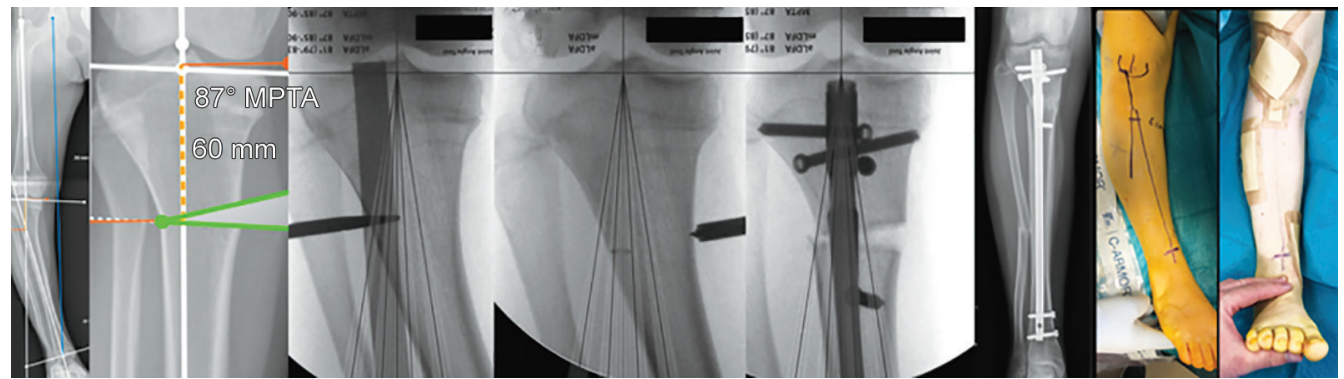

Fig. 4: Proximal tibial osteotomy in a girl with rickets demanding a permanent intramedullary fixation. MPTA, medial proximal tibial angle
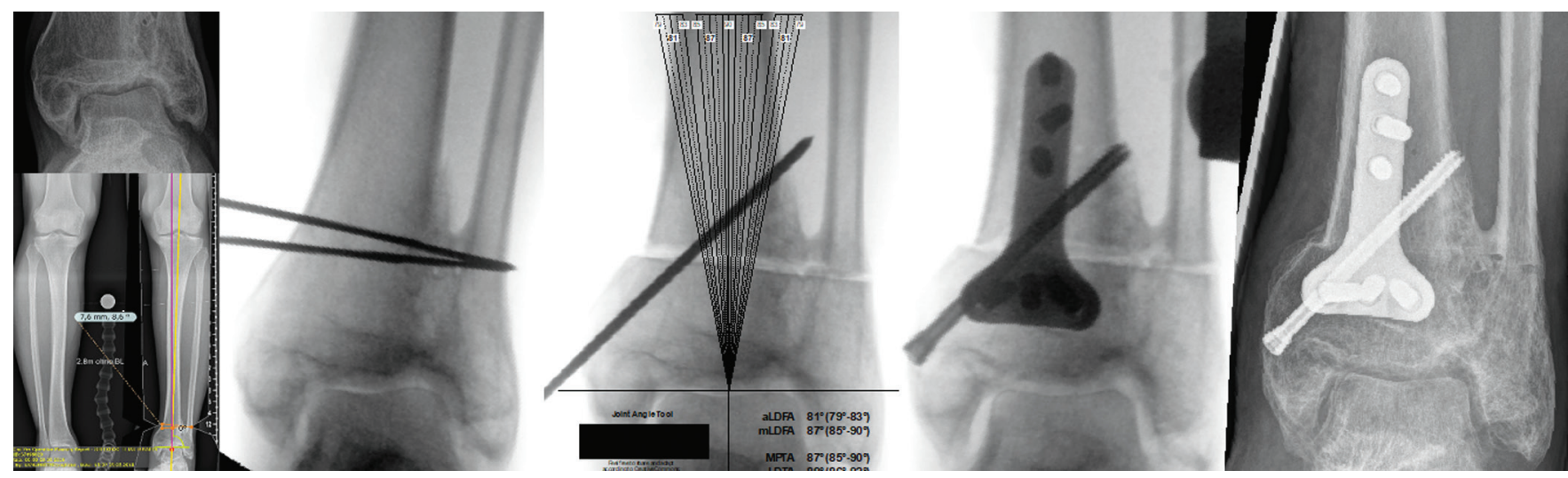

Fig. 5: Supramalleolar osteotomy realigning the anatomic axis

LiveSciences, New Jersey, USA) and a 6-hole locking plate (2.8 mm Flexit, Neosteo, Nantes, France).

\section{Discussion}

Intraoperative options to assess the anatomic axis of the lower limb and execute during reconstructive and trauma surgery are limited. The JAT may prove beneficial in corrective osteotomies around the knee and ankle regardless of the fixation method because of its simplicity. We find it especially helpful and reassuring when correcting coronal and torsional malalignment at the same time and when double osteotomies are necessary. Moreover, the JAT is useful when correcting deformities and limb length with intramedullary devices. Here, the execution of the preoperative plan relies mainly on measuring the distance from the joint line to the level of osteotomy using fluoroscopy. Consequently, the surgeon may encounter several fluoroscopy related obstacles potentially leading to inaccurate execution of the preoperative plan, and thus postoperative malalignment. Often a ruler is intraoperatively placed on the leg to measure the distance from the joint line to the desired place of the osteotomy (Fig. 4). Soft tissue inevitably interposes between the ruler and the bone introducing a source of measuring error due to the divergence of the radiographic beam. It is thus uncertain whether the level of osteotomy is indeed the pre-planned level. ${ }^{12,17}$ As the JAT relies on angles rather than distances, the fluoroscopic magnification does not interfere with its use. The pre-printed joint orientation angles and the long lines of the goniometer ease the accurate intraoperative assessment compared to regular goniometers.
Most other techniques intended to ensure coronal alignment are either complex, expensive, or only applicable at the end of surgery..$^{10-13,15,18}$ These techniques determine the mechanical and anatomic axes using fluoroscopic markers and are thus subject to distortion of the fluoroscope. When applying a radiopaque axis board under the patient during surgery the distortion affects the bone and board similarly but requires a more complex patient positioning during surgery. The fluoroscopic distortion may also contribute to the inaccurate measure from the joint to the planned level of osteotomy. ${ }^{16}$

Unlike most other techniques, the JAT can be applied at all stages of the surgery to achieve and monitor the planned anatomic axis, that is when inserting a guidewire and reaming the canal up to the planned osteotomy level in addition to the end of surgery when the nail has been inserted. This allows enhancement during the surgical steps, for example by changing the placement of the guidewire to achieve the correct level of osteotomy and obtain the desired alignment.

The tool provides additional intraoperative reassurance to the surgeon when inserting expensive medical devices, such as lengthening or bone transport nails. ${ }^{17-20}$ However, it can also be useful when treating fractures or performing corrective osteotomies around the knee and distal tibia. The advantages of the JAT include simplicity, free availability facilitating its application regardless of the clinical setting, and economic resources.

\section{Limitations}

The JAT is applied on the screen of the image intensifier and is therefore not affected by the distortion of the fluoroscope. 
However, a discrepancy between the distorted image of the bone and the JAT may still occur.

Care should be taken, when applying the tool in children under 8 years of age as normal values of the joint orientation angles differ from the adult population. ${ }^{21}$ However, the tool may still be useful in this immature population to control that the intraoperative anatomic axis adheres to the preoperative plan.

The JAT only assesses the coronal plane and does not convey information about the sagittal and axial plane.

Here, we demonstrate the application of JAT in a case series. Further scientific evaluation of its accuracy and clinical value is recommended.

The authors recommend the JAT as an adjunct to other methods assessing coronal alignment intraoperatively. Trauma surgeons, limb lengthening and reconstructive surgeons, knee surgeons, and foot and ankle surgeons may appreciate the additional reassurance that the preoperative plan is executed during the entire surgery.

\section{Conclusion and Clinical Significance}

JAT is a low-cost goniometer providing surgeons intraoperative information about the anatomic axis based on true anteroposterior radiographs. It is applicable throughout the entire surgical procedure irrespective of the method of internal fixation. Thus, it provides additional intraoperative reassurance regarding the coronal alignment of the lower limb. JAT is especially helpful and reassuring when correcting coronal and torsional malalignment at the same time and when double osteotomies are necessary.

JAT is available for free download and thus accessible for everyone. It should be printed on a transparent plastic sheet and laminated for intraoperative use.

\section{References}

1. Paley D. Principles of Deformity Correction. Berlin, Heidelberg: Springer Berlin Heidelberg; 2002.

2. Moreland JR, Bassett LW, Hanker GJ. Radiographic axial analysis of the lower of the extremity. J. Bone Joint Surg Am 1987;69(5):745-749.

3. Fragomen AT, Rozbruch SR. Lengthening and deformity correction about the knee using a magnetic internal lengthening nail. SICOT J 2017;3:25. DOI: 10.1051/sicotj/2017014.

4. Brinkman J-M, Lobenhoffer P, Agneskirchner JD, et al. Osteotomies around the knee: patient selection, stability of fixation and bone healing in high tibial osteotomies. J Bone Joint Surg $\mathrm{Br}$ 2008;90(12):1548-1557. DOI: 10.1302/0301-620X.90B12.21198.

5. Frommer A, Rödl R, Gosheger G, et al. Application of motorized intramedullary lengthening nails in skeletally immature patients: indications and limitations. Unfallchirurg 2018;121(11):860-867. DOI: 10.1007/s00113-018-0541-4.

6. Ashfaq K, Fragomen AT, Nguyen JT, et al. Correction of proximal tibia varus with external fixation. J Knee Surg 2012;25(5):375-384. DOI: 10.1055/s-0031-1299659.
7. Baumgart R. The reverse planning method for lengthening of the lower limb using a straight intramedullary nail with or without deformity correction. Oper Orthop Traumatol 2009;21(2):221-233. DOI: 10.1007/s00064-009-1709-4.

8. Lee DC, Byun SJ. High tibial osteotomy. Knee Surg Relat Res 2012;24(2):61-69. DOI: 10.5792/ksrr.2012.24.2.61.

9. Krettek C, Miclau T, Grun O, et al. Intraoperative control of axes, rotation and length in femoral and tibial fractures. Technical note. Injury 1998;29(Suppl 3):C29-C39. DOI: 10.1016/s00201383(98)95006-9.

10. Hankemeier S, Gosling T, Richter M, et al. Computer-assisted analysis of lower limb geometry: higher intraobserver reliability compared to conventional method. Comput Aided Surg 2006;11(2):81-86. DOI: 10.3109/10929080600628985.

11. Liodakis E, Kenawey M, Liodaki E, et al. The axis-board: An alternative to the cable technique for intraoperative assessment of lower limb alignment. Technol Health Care 2010;18(3):165-171. DOI: 10.3233/ THC-2010-0579.

12. Jacquet C, Chan-Yu-Kin J, Sharma A, et al. More accurate correction using "patient-specific" cutting guides in opening wedge distal femur varization osteotomies. Int Orthop 2019;43(10):2285-2291. DOI: 10.1007/s00264-018-4207-1.

13. Rogers MJ, McFadyen I, Livingstone JA, et al. Computer hexapod assisted orthopaedic surgery (CHAOS) in the correction of long bone fracture and deformity. J Orthop Trauma 2007;21(5):337-342. DOI: 10.1097/BOT.0b013e3180463103.

14. Rudin S, Bednarek DR, Wong R. Accurate characterization of image intensifier distortion. Med Phys 1991;18(6):1145-1151. DOI: 10.1118/1.596623.

15. Boduch A, Hennrikus M, Adebayo T, et al. Accuracy of C-arm measurements in assessment of paediatric femoral fracture shortening. J Child Orthop 2017;11(6):460-464. DOI: 10.1302/18632548.11.170116.

16. Kummer FJ, Grant AM. Possible errors in pin insertion positions using the C-arm. Bull Hosp Jt Dis 2004;62(1-2):67-68.

17. Kold S, Christensen KS. Bone transport of the tibia with a motorized intramedullary lengthening nail-a case report. Acta Orthop 2014;85(2):211-213. DOI: 10.3109/17453674.2014.887953.

18. Horn J, Hvid I, Huhnstock S, et al. Limb lengthening and deformity correction with externally controlled motorized intramedullary nails: evaluation of 50 consecutive lengthenings. Acta Orthop 2019;90(1):81-87. DOI: 10.1080/17453674.2018.1534321.

19. Calder PR, McKay JE, Timms AJ, et al. Femoral lengthening using the Precice intramedullary limb-lengthening system: outcome comparison following antegrade and retrograde nails. Bone Joint J 2019;101-B(9):1168-1176. DOI: 10.1302/0301-620X.101B9.BJJ-20181271.R1.

20. Olesen UK, Nygaard T, Prince DE, et al. Plate-assisted bone segment transport with motorized lengthening nails and locking plates. J Am Acad Orthop Surg Glob Res Rev 2019;3(8):e064. DOI: 10.5435/ JAAOSGlobal-D-19-00064.

21. Sabharwal S, Zhao C, Edgar M. Lower limb alignment in children: reference values based on a full-length standing radiograph. J Pediatr Orthop 2008;28(7):740-746. DOI: 10.1097/ BPO.0b013e318186eb79. 